\title{
An objective measure of REM activity
}

\author{
RICHARD J. McPARTLAND, BRIAN L. WEISS, and DAVID J. KUPFER* \\ Department of Psychiatry, Western Psychiatric Institute and Clinic \\ University of Pittsburgh School of Medicine, Pittsburgh, Pennsylvania 15261
}

\begin{abstract}
Recent emphasis on REM sleep abnormalities has begun to yield significant differences between "normals" and patients with affective syndromes or schizophrenia. In our efforts to objectively "quantitate aspects of REM sleep during all-night studies in psychiatric patients, we have developed the "REM analyzer" for automatic on-line measurement. As a practical application of the REM analyzer, 20 psychiatric patients (12 inpatients and 8 outpatients) were studied. Both cross-sectional and longitudinal-sleep studies were obtained; predrug and drug periods were also compared. From the REM analyzer, the number of rapid eye movements during each REM period $\left(C_{n}\right)$ and the sum of their integrals during each REM period $\left(I_{n}\right)$ were recorded. The ratio I/C was named "REM weight" (the "average rapid-eye-movement area"). The objective measures of REM sleep which highly correlate with a number of visual methods of defining REM sleep and with diagnostic and therapeutic implications in affective and schizophrenic disorders are discussed in this paper. The method appears particularly suitable for the differentiation of tonic and phasic components of REM sleep and, thus, for the investigation of REM suppression-compensation models.
\end{abstract}

The study of REM sleep, along with other sleep-pattern changes observed in psychiatric syndromes, has already yielded significant findings in a number of areas. Despite certain differences in sleep-pattern changes noted between unipolar and bipolar depressions (Kupfer et al, 1972), the findings that (1) shortened REM latency (time to the onset of the first REM period) is characteristic in all depressive syndromes (Kupfer \& Foster, 1972), and (2) a severe reduction in REM sleep in schizophrenia is not followed by the degree of REM compensation found in normals or patients with affective syndromes (Snyder, 1969; Kupfer et al, 1970) suggest that REM sleep changes represent a promising area for further investigation.

The problem of how the frequency and type of rapid eye movement can best be measured has remained perlexing. Although some investigators have measured "REM activity" by visually scoring REM intensity on a 0-8 scale for each minute (Kupfer \& Heninger, 1972), and others have used 10- or 20-sec REM-containing epochs (Salzarulo, 1972; Coulter, 1971; DeBarros-Ferreira, 1971), to date the only accepted method has been to total REM time by summing up all 60-sec REM-containing epochs (Rechtschaffen \& Kales, 1968). All these visual methods. of scoring have the major limitations of being only imprecise measures of REM time and intensity and of being subject to interrater variabilities.

In an effort to overcome these limitations, our laboratory has designed an instrument for automatic on-line measurement of several REM parameters. This instrument, the "REM analyzer" [details of which have been given elsewhere (McPartland, Kupfer, \& Foster,

*Address requests for reprints to Dr. Kupfer, Department of Psychiatry, University of Pittsburgh School of Medicine, Western Psychiatric Institute and Clinic, 3811 O'Hara Street, Pittsburgh, Pennsylvania 15261
1973)], has the capacity to measure the integral, $\mathrm{I}=$ SAdt, where $\mathrm{I}$ is the integral $(1$ unit equals 17.2 microV sec), $\mathrm{A}$ is the amplitude, and $\mathrm{t}$ is the time, of each REM waveform (see Fig. 1). It can also count the number of REMs (C) in any predetermined time interval. This report deals with the first clinical application of the REM analyzer.

\section{METHOD}

The study included sleep data from 20 drug-free psychiatric patients and data from a subgroup of 11 patients who subsequently received psychotropic drugs. Both inpatients (12) and outpatients(8) were studied on the clinical-research ward. The inpatients slept in their own hospital rooms, while the outpatients slept in a special room in the research ward. There were 10 males and 10 females, with ages ranging from 22 to 63 years (mean age 38.1 years). Each patient was diagnosed independently by three senior psychiatrists, utilizing an extensive diagnostic interview and a set of patient self-rating forms (Kupfer \& Detre, 1971). The diagnostic breakdown of the total sample included: 11 depressives, 4 schizophrenics, and 5 neurotics. The sleep of $20 \mathrm{Ss}$ was studied over at least 2 consecutive nights, with continuous recordings of their electroencephalogram (EEG), electrooculogram (EOG), and submental electromyogram (EMG). To avoid possible adaptation effects, data from the second night only was used in this study. None of the patients had received any medication for at least 2 weeks prior to the investigation. In the second drug subgroup study, 7 of the inpatients were studied, both prior toand following the administration of psychotropic drugs. The depressed patients were given amitriptyline; the schizophrenic patients received trifluoperazine. Following adaptation, two to four records were obtained both during the predrug period and again 7 to 10 days after patients began receiving active medication.

All sleep records were evaluated according to the standardized techniques (Rechtschaffen \& Kales, 1968) by a scorer unaware of the patient's sleep habits, clinical status, and data obtained from the REM analyzer. Each minute of REM sleep was scored visually on a $0-8$ scale for rapid-eye-movement patterns; the sum for each REM period provided a measure of "REM activity" for that period. The temporal position and extent of each REM 


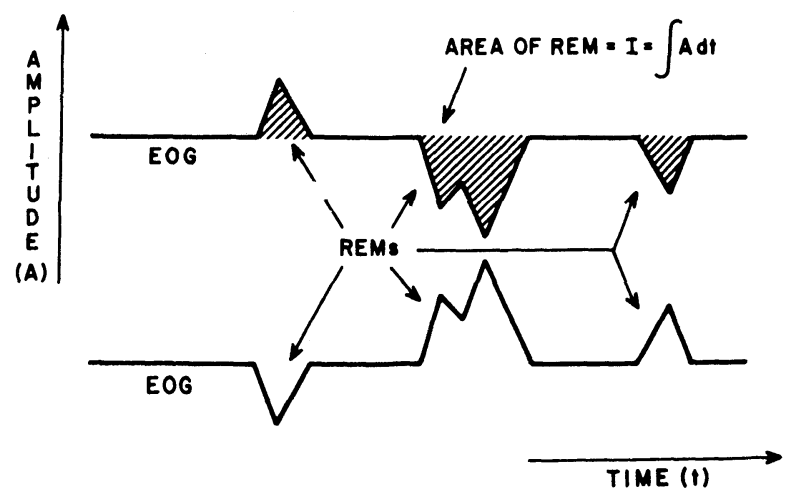

Fig. 1. Left and right EOG tracings as displayed by a polygraph. Shaded areas are the integrals of the individual REMs. Their sum is the total REM integral for the corresponding time period.

period was determined by the scorer, thus ensuring that the same time intervals were scored for REM parameters, both visually and automatically. From the REM analyzer, the number of rapid eye movements during each REM period $\left(C_{n}\right.$, where $n$ represents the first, second, third, fourth, or fifth REM period), and the sum of their integrals during each REM period $\left(I_{n}\right)$, was recorded. The ratio $I / C$ (or for individual REM periods $I_{n} / C_{n}$ ), where $I$ is the sum of all the $I_{n}$ s of the night and $C$ is the similar sum for $C_{n}$, was named "REM weight" (the "average rapid-eye-movement area"). Certain REM sleep features (REM sleep time, RT; REM activity, RA; REM integral, I; REM count, C; REM weight, I/C) in the 20 patients were then compared, using Pearsonian correlations.

Drug-free REM sleep data, in the seven depressed and four schizophrenic inpatients, were compared with data from the period when the patients were receiving medication. The mean and standard error for each REM parameter was obtained for both predrug and drug REM periods by grouping together and averaging individual REM period values. Since the number of REM periods varied with respect to each S, REM data for any particular night was not used in our calculations unless the patient had had at least three REM periods (only one night of REM data was discarded). $T$ tests were used to compare data from these inpatients.

\section{RESULTS}

As shown in Table 1, certain all-night-REM sleep parameters from 20 psychiatric patients correlate significantly with each other. Of particular interest is that REM sleep time (RT) correlates significantly with REM activity (RA), REM integral (I) and REM count (C); REM activity with $I$ and $C$; and both the integral (I) and count (C) with the RA and RT. Because the integral significantly correlates at a high level with the count, correlation between the REM weight ratio (I/C) and other parameters is necessarily low.

Psychotropic drug effects were examined by comparing mean REM period values of integral, count, and REM weight (I/C ratio) for the schizophrenic subgroup and the depressive subgroup. As shown in
Table 2, the average REM weight per REM period of the schizophrenic group prior to medication differed significantly from both the nonmedicated depressive group as well as from the medicated schizophrenic group. No such changes were noted in the depressive group.

Reliability of the REM analyzer was tested by recording REM sleep EOG data for four Ss on magnetic recording tape and replaying the data to the REM analyzer twice. Variations of the count and integral between passes for the same $\mathrm{S}$ were always less than $3 \%$.

\section{DISCUSSION}

This report represents the first clinical application of the REM analyzer in our effort to objectively quantitate aspects of REM sleep. The REM analyzer measures of I (integral) and C (count) correlated highly with REM time and REM activity, parameters derived from the visual method of measurement. These correlations indicate the validity of this automated method. Reliability of the REM analyzer is high, in comparison with human scorers. Agnew and Webb (1972), who have developed an effective yet arduous system for evaluating and maintaining reliability of human scorers, reported an overall scoring reliability of $91 \%$ between four experienced scorers within the same laboratory. Reported average reliability for scoring REM time was $96 \%$. Reports of interlaboratory scoring reliability show even greater deviations (Monroe, 1969); however, REM time was again the least variant parameter reported. Published data is not available on scorer reliability when evaluating REM activity. However, experience in our laboratory indicates that it is more difficult to attain high reliability for REM activity than for REM time. The obvious advantage of automated REM scoring is the availability of the integral and count, two parameters extremely cumbersome to obtain by normal scoring methods. Standardization obtained by using the analyzer would increase the accuracy when comparing results from different laboratories.

Despite the small sample, it appeared that the REM weight of untreated schizophrenic patients is significantly lower than their REM weight when they receive phenothiazines, and significantly lower than

Table 1

Correlations Between Automated and Visual Measures of REM Sleep

\begin{tabular}{lllll}
\hline RT & & & & \\
RA & $0.77 * *$ & & & \\
I & $0.69 * *$ & $0.86 * *$ & & \\
C & $0.69 * *$ & $0.86 * *$ & $0.90 * *$ & \\
I/C & 0.22 & 0.34 & $0.51^{*}$ & 0.12 \\
& RT & RA & I & C \\
\hline & $* p<.05$ & & $* * p<.001$
\end{tabular}


Table 2

Mean REM Parameters Per REM Period

\begin{tabular}{|c|c|c|c|c|c|c|}
\hline \multirow[b]{2}{*}{ REM Sleep Parameters } & \multicolumn{2}{|c|}{ Schizophrenics } & \multirow[b]{2}{*}{$\mathrm{p}$} & \multicolumn{2}{|c|}{ Depressives } & \multirow[b]{2}{*}{$\mathrm{p}$} \\
\hline & Predrug & Drug & & Predrug & Drug & \\
\hline REM Time & $23.7 \pm 0.7$ & $20.3 \pm 0.5$ & n.s. & $18.9 \pm 0.4$ & $19.5 \pm 0.9$ & n.s. \\
\hline REM Activity & $48.1 \pm 1.7$ & $42.8 \pm 1.3$ & n.s. & $46.5 \pm 1.5$ & $57.1 \pm 3.3$ & n.s. \\
\hline Integral (I) & $90.3 \pm 3.7$ & $93.8 \pm 3.8$ & n.s. & $104.7 \pm 4.1$ & $142.7 \pm 11.2$ & n.s. \\
\hline Count $(C)$ & $154.6 \pm 6.6$ & $116.1 \pm 3.9$ & n.s. & $132.5 \pm 4.2$ & $177.9 \pm 11.1$ & n.s. \\
\hline REM Weight (I/C Ratio) & $0.57 \pm 0.03^{*}$ & $0.77 \pm 0.04$ & .001 & $0.71 \pm 0.04$ & $0.71 \pm 0.05$ & n.s. \\
\hline
\end{tabular}

${ }^{*} p<.05$ for $I / C$ ratio between schizophrenic predrug and depressive predrug groups.

depressed patients, either before or during drug treatment with tricyclic antidepressants. If this phenomenon is confirmed in a larger group of schizophrenic patients, it may represent one way of distinguishing REM sleep in schizophrenics from REM sle $p$ in other patients. This weak nocturnal rapid-eye-movement activity is in contrast to the higher-than-normal eye activity exhibited by schizophrenics during daytime eye-tracking tasks (Holzman et al, 1973), an increase attributed to perceptual dysfunction. These findings raise the question of whether a shift in eye-movement activity from the dreaming to the awake periods occurs in schizophrenic patients.

One application of the REM analyzer will be in the investigation of REM suppression-compensation phenomena. Recently, two types of REM suppression have been defined: Type 1 is marked by changes in the REM-to-REM cycle length, followed by REM rebound on drug withdrawal; Type 2 is a function of decreased length of each REM period, rather than a lengthening of the REM-to-REM cycle, and is unassociated with REM rebound (Kupfer et al, 1974). For example, our preliminary investigation with amitriptyline indicated that Type 1 suppression may be associated with overall decreased REM time, but not with significant changes in the REM integral or count measures. Although during the drug period, REM latency was markedly prolonged, REM cycle length tended to be longer, and the number of REM periods was reduced; REM weight showed no change per REM period, despite the alteration in REM "location" during the night. Further work in this area is currently under way in our laboratory.

\section{REFERENCES}

Agnew, H. W., Jr., \& Webb, W. B. Sleep stage scoring. Abstracted in JSAS catalog of Selected Documents in Psychology, 1972, 2,56 .

Coulter, J. D., Lester, B. K., \& Williams, H. L. Reserpine and sleep. Psychopharmacologia, 1971, 19, 134-147.

DeBarros-Ferreira, M. An approach to a quantitative study of eye movements during paradoxical sleep in a clinical setting. Electroencephalography \& Clinical Neurophysiology, 1971, 30, 248-254

Holzman, P. S., Proctov, L. R., \& Hughes, D. W. Eye tracking patterns in schizophrenia. Science, 1973, 181, 179-181.

Kupfer, D. J., \& Detre, T. The development and application of the KDS-1 in inpatient and outpatient settings. Psychological Reports, 1971, 29, 607-617.

Kupfer, D. J., \& Foster, F. G. Interval between onset of sleep and rapid eye movement sleep as an indicator of depression. Lancet, 1972, II, 684-686.

Kupfer, D. J., \& Heninger, G. R. REM activity as a correlate of mood changes throughout the night. Archives of General Psychiatry, 1972, 27, 368-373.

Kupfer, D. J., Himmelhoch, J., Swartzburg, M., Anderson, C. M., Byck, R., \& Detre, T. Hypersomnia in manic-depressive disease. Diseases of the Nervous System, 1972, 33, 720-724.

Kupfer, D. J., Reynolds, C. F., Weiss, B. L., \& Foster, F. G. Lithium carbonate and sleep in affective disorders; Further considerations. Archives of General Psychiatry, 1974, 30, 79-84.

Kupfer, D. J., Wyatt, R. J., Scott, J., \& Snyder, F. Sleep dist urbance in acute schizophrenic patients. American Journal of Psychiatry, 1970, 126, 47-57.

McPartland, R. J., Kupfer, D. J., \& Foster, F. G. Rapid eye movement analyzer. Electroencephalography \& Clinical Neurophysiology, 1973, 34, 317-320.

Monroe, L. Inter-rater reliability and role of experience in scoring EEG sleep records: Phase I. Psychophysiology, 1969, $5,376-384$.

Rechtschaffen, A., \& Kales, A. (Eds.), A manual of standard terminology, techniques and scoring system for sleep stages of human subjects, (Public Health Service) Washington: U.S Government Printing Office, 1968.

Salzarulo, P. Variations with time of the quantity of eye movements during fast sleep in man. Electroencephalography \& Clinical Neurophysiology, 1972, 32, 409-416.

Snyder, F. Dynamic aspects of sleep disturbance in relation to mental illness. Biological Psychiatry, 1969, 1, 119-130.

(R eceived for publication February 21, 1974; revision received June 6,1974 .) 